The Astrophysical Journal, 672: L9-L12, 2008 January 1

(C) 2008. The American Astronomical Society. All rights reserved. Printed in U.S.A.

\title{
STARBURST GALAXIES IN CLUSTER-FEEDING FILAMENTS UNVEILED BY SPITZER
}

\author{
DARIO FADDA ${ }^{1}$ \\ NASA Herschel Science Center, California Institute of Technology, Mail Code 100-22, Pasadena, CA 91125; fadda@ipac.caltech.edu \\ ANDREA BIVIANO ${ }^{2}$ \\ INAF-Osservatorio Astronomico di Trieste, via G. B. Tiepolo 11, I-34143 Trieste, Italy \\ Francine R. Marleau and Lisa J. Storrie-Lombardi \\ Spitzer Science Center, California Institute of Technology, Mail Code 220-6, Pasadena, CA 91125 \\ AND \\ FLORENCE DURRET \\ Institut d'Astrophysique de Paris, CNRS, Université Pierre et Marie Curie, 98bis Boulevard Arago, F-75014 Paris, France \\ Received 2007 October 8; accepted 2007 November 14; published 2007 December 4
}

\begin{abstract}
We report the first direct detection with Spitzer of galaxy filaments. Using Spitzer and ancillary optical data, we have discovered two filamentary structures in the outskirts of the cluster Abell 1763. Both filaments point toward Abell 1770, which lies at the same redshift as Abell $1763(z=0.23)$, at a projected distance of $\sim 13$ Mpc. The X-ray cluster emission is elongated along the same direction. Most of the far-infrared emission is powered by star formation. According to the optical spectra, only one of the cluster members is classified as an active galactic nucleus. Star formation is clearly enhanced in galaxies along the filaments: the fraction of starburst galaxies in the filaments is more than twice than that in other cluster regions. We speculate that these filaments are feeding the cluster Abell 1763 by the infall of galaxies and galaxy groups. Evidence for one of these groups is provided by the analysis of galaxy kinematics in the central cluster region.
\end{abstract}

Subject headings: galaxies: clusters: individual (Abell 1763) — galaxies: kinematics and dynamics infrared: galaxies

\section{INTRODUCTION}

Thanks to large redshift surveys, it is presently well established that baryonic matter is arranged in the universe in a network of voids and filamentary structures (e.g., de Lapparent et al. 1986; Doroshkevich et al. 2001; Pimbblet et al. 2004). Galaxy clusters are found at the intersections between filaments. Hierarchical models of galaxy formation (e.g., Benson 2005) and observations (e.g., Zabludoff \& Franx 1993; Ceccarelli et al. 2005; Cortese et al. 2006) show that clusters constantly accrete small galaxy groups through filaments. These accreted galaxies undergo an accelerated evolution when they meet the denser cluster environment, experiencing a short starburst phase followed by the quenching of their star formation activity as their gas reservoirs are consumed and/or stripped off (e.g., Bekki 1999; Balogh et al. 2000; Fujita 2004). These events are traced by differences in morphology and star formation rate of galaxies inside and outside clusters (e.g., Dressler 1980; Balogh et al. 1998; Goto et al. 2003; Kodama et al. 2004).

Much of what we have learned so far about the evolution of galaxies in clusters comes from detailed studies of the inner cluster regions, where the contrast with respect to the field is high, so that most spectroscopically targeted galaxies have a high probability of being cluster members. We are therefore limited to studying the later stages of the environmental processes that drive galaxy evolution in clusters. We can follow

\footnotetext{
${ }^{1}$ Visiting Astronomer, Kitt Peak National Observatory, National Optical Astronomy Observatory, which is operated by the Association of Universities for Research in Astronomy, Inc. (AURA), under cooperative agreement with the National Science Foundation.

${ }^{2}$ Visiting Astronomer, Italian Telescopio Nazionale Galileo (TNG), operated on the island of La Palma by the Fundación Galileo Galilei of the INAF (Istituto Nazionale di Astrofisica) at the Spanish Observatorio del Roque de los Muchachos of the Instituto de Astrofisica de Canarias.
}

the entire evolution of these galaxies, only observing the external regions where infalling field galaxies first encounter the cluster potential and the intracluster gas. Unfortunately, studies of this kind are extremely rare since they require wide-field images coupled with intensive spectroscopic follow-up observations (see, e.g., Porter \& Raychaudhury 2007).

Moreover, in order to better characterize galaxy properties through their spectral energy distributions (SEDs), multiwavelength observations are needed. Until recently, most studies of the external regions of galaxy clusters have been based on optical/near-infrared data (see, e.g., Treu et al. 2003; Gerken et al. 2004; Rines et al. 2005). On the other hand, infrared studies of clusters have been limited to the inner regions (see the review of Metcalfe et al. 2005). With the advent of Spitzer (Werner et al. 2004), the outskirts of galaxy clusters have now started to be observed (Bai et al. 2006, 2007; Geach et al. 2006; Marcillac et al. 2007).

In this Letter we report our discovery with Spitzer of two galaxy filaments in the outer region of the cluster Abell 1763 (at a redshift $\bar{z}=0.23$ ). The two filaments are rich in actively star-forming galaxies, which are probably exhausting their gas supply while entering the virialized cluster environment. Throughout this paper we use $H_{0}=70 \mathrm{~km} \mathrm{~s}^{-1} \mathrm{Mpc}^{-1}$, $\Omega_{m}=0.3, \Omega_{\Lambda}=0.7$.

\section{SPITZER AND ANCILLARY OBSERVATIONS}

Abell 1763 was surveyed with the InfraRed Array Camera (IRAC; Fazio et al. 2004) and the Multiband Imaging Photometer for Spitzer (MIPS; Rieke et al. 2004) as part of a program to observe three medium-redshift clusters up to large distances from the center study the star formation activity in infalling galaxies. A field of $40^{\prime} \times 55^{\prime}$ was observed with MIPS at 24, 70 , and $160 \mu \mathrm{m}$. An overlapping field of $39^{\prime} \times 39^{\prime}$ was observed at 3.6, 4.5, 5.8, and $8.0 \mu \mathrm{m}$ with IRAC. The entire field 
TABLE 1

Redshifts Measured

\begin{tabular}{|c|c|c|c|c|}
\hline \multirow[b]{2}{*}{ Survey } & \multicolumn{3}{|c|}{$N_{z}$} & \multirow[b]{2}{*}{ Flux Limits } \\
\hline & Total & Cluster & Cluster and $24 \mu \mathrm{m}$ & \\
\hline WIYN ....... & 360 & 80 & 80 & $\begin{array}{c}r^{\prime}<20.5 \\
f_{24}>0.3 \mathrm{mJy}\end{array}$ \\
\hline TNG . & 157 & 101 & 34 & $r^{\prime}<21.5$ \\
\hline SDSS . & 96 & 18 & 11 & $r^{\prime}<20.8$ \\
\hline Total .......... & 581 & 181 & 106 & $\ldots$ \\
\hline
\end{tabular}

is covered by the Sloan Digital Sky Survey (SDSS) in the $u^{\prime}, g^{\prime}, r^{\prime}, i^{\prime}$, and $z^{\prime}$ bands. To detect fainter objects in preparation for the spectroscopic follow-up observations, we obtained an $r^{\prime}$ image of the $24 \mu \mathrm{m}$ field with the Large Format Camera on the Palomar 200 inch telescope, reaching 2 mag deeper than the SDSS image. We obtained 382 spectra with the Hydra spectrograph on the WIYN telescope at Kitt Peak for most of the sources brighter than $0.3 \mathrm{mJy}$ at $24 \mu \mathrm{m}$, corresponding to an average signal-to-noise ratio of 5 , with optical counterparts brighter than $r^{\prime}=20.5$. To obtain a better description of the velocity distribution of the cluster, we obtained 198 additional spectra mainly in the central region with the DOLORES multiobject spectrograph on the TNG telescope in La Palma, Spain. In this case, we targeted fainter galaxies with $r^{\prime}<21.5$ including faint infrared sources and early-type galaxies. Finally, to obtain estimates of the stellar mass of the galaxies as well as a better description of their SEDs, we obtained $J, H$, and $K_{s}$ images of most of the $24 \mu \mathrm{m}$ field with the Wide InfraRed Camera on the Palomar 200 inch telescope. These images are typically 3 mag deeper than the 2MASS (Skrutskie et al. 2006) images in this field. The data and their analysis will be presented in forthcoming papers.

\section{SPATIAL AND REDSHIFT DISTRIBUTIONS}

The number of redshifts from our WIYN and TNG observations, as well as that from SDSS, are reported in Table 1. Cluster members were identified using the procedure developed by den Hartog \& Katgert (1996). The spatial distribution of the selected cluster members (Fig. 1) presents a remarkable asymmetry outside $r_{500}(\sim 1.5 \mathrm{Mpc})$, the radius at which the mean interior overdensity is 500 times the critical density. We computed $r_{500}$ using the relationship by Carlberg et al. (1997) between $r_{200}$ and velocity dispersion and a Navarro, Frenk, \& White (1997) profile with concentration $c=4$. The asymmetry is caused by the presence of two galaxy filaments oriented at $\sim 51^{\circ}$ and $\sim 73^{\circ}$ counterclockwise from north, hereafter referred to as the northern $(\mathrm{N})$ and southern $(\mathrm{S})$ filaments, respectively. The $\mathrm{S}$ (and perhaps also the $\mathrm{N}$ ) filament is directed toward the neighboring cluster Abell 1770, located at approximately the same redshift as Abell $1763(\bar{z}=0.2313)$, but $\sim 13 \mathrm{Mpc}$ away. The $\mathrm{N}$ and $\mathrm{S}$ filaments have different mean velocities: -135 \pm 138 and $300 \pm 99 \mathrm{~km} \mathrm{~s}^{-1}$, respectively, relative to the cluster mean velocity, suggesting that the two filaments are entering the cluster from opposite directions (see Fig. 2).

Within $r_{500}$, the filaments are no longer distinguishable from the inner cluster. However, the galaxy distribution in the inner region and the X-ray-emitting intracluster gas are elongated along roughly the same direction of the two filaments. The Xray image also shows an emission tail southwest of the center which leads Lima-Neto \& Durret (2007) to suggest that Abell 1763 is undergoing accretion events. Moreover, the X-ray temperature map conserves traces of a previous merger from the southeast (Prokhorov \& Durret 2007). An ongoing accretion

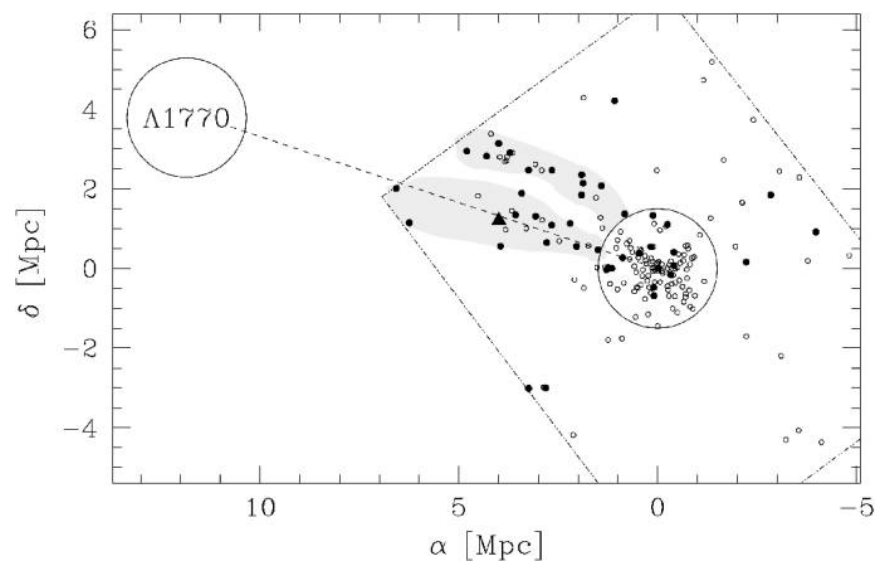

FIG. 1.-Spatial distribution of the 181 spectroscopically selected members of the cluster Abell 1763. Target selection was done exclusively with $24 \mu \mathrm{m}$ sources in the region outside the $1.5 \mathrm{Mpc}$ radius central region. In the inner region, early-type galaxies were targeted also to better describe the velocity distribution of the cluster. The dotted rectangle corresponds to the $24 \mu \mathrm{m}$ MIPS field. Starburst galaxies and AGNs are marked with filled dots and triangles, respectively. Circles of $1.5 \mathrm{Mpc}$ radius indicate the centers of Abell 1763 and Abell 1770, connected by a dashed line. The shaded regions have been traced by eye to highlight two filamentary structures. In these regions there is a clear excess of starburst galaxies.

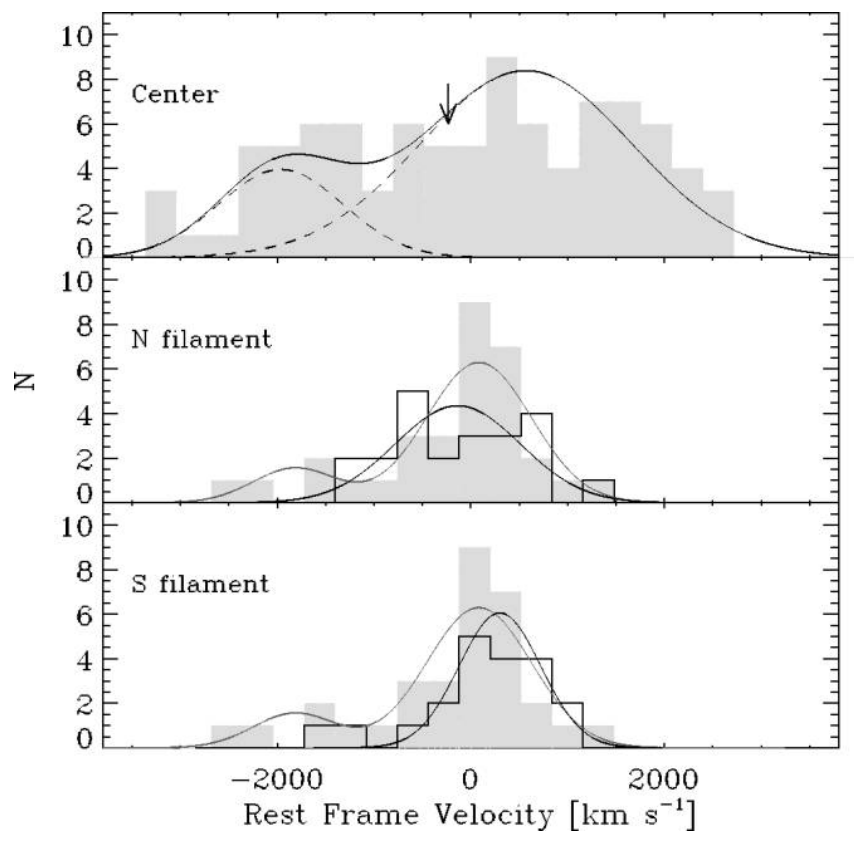

FIG. 2.-Rest-frame velocity distributions for different cluster regions. Top: Galaxies within $1.5 \mathrm{Mpc}$ of the X-ray cluster center. The solid line represents a two-Gaussian fit to the velocity distribution, and the dashed lines represent the two separate components of the fit, with velocity dispersions of $641 \pm$ $108 \mathrm{~km} \mathrm{~s}^{-1}$ (left) and $1097 \pm 152 \mathrm{~km} \mathrm{~s}^{-1}$ (right). The vertical arrow indicates the BCG velocity. Middle and bottom: Galaxies outside $1.5 \mathrm{Mpc}$. The shaded histogram shows the velocity distribution of galaxies outside the filaments. The gray line represents a fit to this velocity distribution with two Gaussians with velocity dispersions of $431 \pm 183 \mathrm{~km} \mathrm{~s}^{-1}$ (left) and $543 \pm 104 \mathrm{~km} \mathrm{~s}^{-1}$ $(r i g h t)$. The solid-line histograms represent the velocity distributions of galaxies in the $\mathrm{N}$ filament (middle) and S filament (bottom). The solid lines represent robust Gaussian fits to the filament velocity distributions, with velocity dispersions of $645 \pm 92 \mathrm{~km} \mathrm{~s}^{-1}$ and $422 \pm 59 \mathrm{~km} \mathrm{~s}^{-1}$ (N filament and $\mathrm{S}$ filament, respectively). Errors on the velocity dispersions are $1 \sigma$, based on 1000 bootstrap resamplings. 
scenario is reinforced by the radio observations of the brightest cluster galaxy (BCG), which is a wide-angle tail source with bent radio emission (Lima-Neto \& Durret 2007). The bending is probably caused by the pressure of the intracluster gas as the BCG moves relative to it, heading toward Abell 1770. Although the direction of motion is opposite to what would be naively expected if the filaments were feeding the cluster, it can be explained as the result of a non-dissipative collision with an infalling group reaching the cluster from the filaments which displaced the BCG from its original location (the galaxy is now $0.1 \mathrm{Mpc}$ off the $\mathrm{X}$-ray peak emission).

Additional supporting evidence for an ongoing merger event in the cluster center comes from the analysis of the velocity distribution of the cluster member galaxies. In the inner region, the velocity distribution deviates from Gaussianity (see Fig. 2). The value of the kurtosis, $K=2.1$, is significantly less than that expected for a Gaussian distribution ( $>99 \%$ confidence level). Such a platykurtic distribution can be interpreted as the superposition of two Gaussians with different means (see Fig. 2). A similar decomposition is obtained also through a non-parametric adaptive-kernel technique (Fadda et al. 1998). The two systems differ in mean velocity by $\sim 2500 \mathrm{~km} \mathrm{~s}^{-1}$, and have velocity dispersions $\sigma_{v} \sim 1100$ and $\sim 650 \mathrm{~km} \mathrm{~s}^{-1}$. These correspond to X-ray temperatures $T_{\mathrm{X}} \sim 7$ and $\sim 2.5 \mathrm{keV}$, respectively, using the $T_{\mathrm{X}}-\sigma_{v}$ relationship (e.g., Girardi et al. 1996). These values are close to the observational estimates by LimaNeto \& Durret (2007) $T_{\mathrm{X}}=7.2 \pm 0.4 \mathrm{keV}$ for the main cluster and $T_{\mathrm{X}}=3.4 \pm 0.4 \mathrm{keV}$ for the detected southwest emission tail. It is therefore tempting to identify the two systems detected in X-ray with the two systems detected in velocity space.

\section{GALAXY PROPERTIES}

We have fitted the SEDs of the 181 spectroscopically identified cluster members using a set of templates built with GRASIL (Silva et al. 1998) which includes elliptical, spiral, starburst, and poststarburst SEDs (Biviano et al. 2004). Using an alternative set of templates (Polletta et al. 2007) we obtained similar results. SEDs of active galactic nuclei (AGNs) were not considered since only one cluster member, which lies on the $\mathrm{S}$ filament (see Fig. 1), is an AGN according to its optical spectrum. Moreover, the sources with IRAC fluxes do not occupy the color diagram region where AGNs are expected (Lacy et al. 2007) and none of the cluster members corresponds to point sources in the $X M M$ image of the field.

Using the best-fit templates we have then determined the galaxy stellar masses $\left(M_{\star}\right)$ and star formation rates (SFRs). The $M_{\star}$ values are determined from the rest-frame $K_{s}$-band luminosities $L_{K}$ by adopting $M_{\star} / L_{K}=1.2$ and 0.5 , for early- and late-type templates, respectively (Lin et al. 2003). SFRs are determined from total infrared luminosities using the relationship of Kennicutt (1998).

We define the ratio $f_{\mathrm{sb}} \equiv \mathrm{SFR} \times \tau_{\mathrm{sb}} / M_{\star}$, where $\tau_{\mathrm{sb}}$ is the timescale over which the galaxy is assumed to form stars at the currently observed SFR. We take $\tau_{\mathrm{sb}}=100 \mathrm{Myr}$ as typical durations of starburst episodes (Barton et al. 2000). High values of $f_{\mathrm{sb}}$ identify galaxies with elevated SFRs given their stellar mass. Hereafter, galaxies with $f_{\mathrm{sb}}>0.25$ are called starburst galaxies. Given that a galaxy gas content is at most $25 \%$ of its total baryonic mass (Young \& Scoville 1991), starburst galaxies will consume their entire gas content in the current starburst episode, if it lasts indeed 100 Myr.

Starburst galaxies preferentially inhabit the two filaments (see Fig. 1). This is confirmed by the following statistical anal- yses, which are performed on the subsample of cluster members with $24 \mu \mathrm{m}$ emission, in order to ensure completeness. A bidimensional Kolmogorov-Smirnov test (Fasano \& Franceschini 1987) indicates that the spatial distribution of starburst galaxies is indeed significantly different from that of other cluster members, ( $>99 \%$ confidence level). Moreover, the fraction of starburst galaxies is $0.6 \pm 0.1,0.3 \pm 0.1$, and $0.2 \pm 0.1$ in the filaments, the central $r_{500}$ region, and the outer region excluding the filaments, respectively. In the same regions, the average $f_{\mathrm{sb}}$ is $0.26 \pm 0.02,0.14 \pm 0.02$, and $0.17 \pm 0.02$, respectively. The distributions of the $f_{\mathrm{sb}}$ values are nearly identical in the two filaments.

\section{DISCUSSION AND CONCLUSIONS}

Using infrared Spitzer photometric data, complemented with optical/near-infrared photometric and spectroscopic data, we have discovered two galaxy filaments leading into the $z=$ 0.23 cluster Abell 1763. The $24 \mu \mathrm{m}$ selection has made the filaments stand out very clearly against the background and the dense cluster region. This is due to the enhanced starforming activity among the filament galaxies, as most of the $24 \mu \mathrm{m}$ emission is of stellar origin (see also Geach et al. 2006; Marcillac et al. 2007).

Both filaments extend in the direction toward the neighboring cluster Abell 1770, which is located $\sim 13 \mathrm{Mpc}$ away. The Abell 1763 intracluster gas is elongated in the same direction, as indicated by its X-ray morphology (Lima-Neto \& Durret 2007). The cluster is probably being fed by the two filaments along which galaxies and galaxy groups accrete onto the main cluster. Evidence for ongoing accretion is provided by the detection of substructures both in the X-ray emitting intracluster gas (LimaNeto \& Durret 2007) and in the velocity distribution of cluster galaxies (see Fig. 2). Since the spatial distribution of the galaxies identified as part of the low-velocity subcluster is not compact, the subcluster-cluster collision has probably occurred already, leading to the disruption of the colliding subcluster. This scenario could explain the displacement of the BCG from the cluster center, and its relative motion with respect to the intracluster gas (Lima-Neto \& Durret 2007).

Galaxies belonging to the two filaments have higher SFRs, on average, than other cluster members, both those in the inner $r_{500}$ region and those outside but not in filaments. We therefore conclude that filaments are environments favorable to the onset of a galaxy starburst activity. Processes related to the dense intracluster gas are not effective in filaments, leaving gravitational interactions among galaxies as the most promising candidate for the stimulation of starbursts. In fact, the relatively high density of galaxies in filaments compared to the general field, and their relatively low velocity dispersion $\left(\sim 500 \mathrm{~km} \mathrm{~s}^{-1}\right)$ compared to the cluster, enhances the tidal effect of galaxy encounters (Mamon 1996) and hence the probability of an induced star-forming activity (Barnes \& Hernquist 1996).

Our results and conclusions find support in recent Spitzer investigations of other clusters. Marcillac et al. (2007) have found that the mid-infrared selected galaxies in a distant cluster $(z=0.83)$ are associated with infalling galaxies. Bai et al. (2007) have suggested that the cluster environment is able to stimulate the star formation activity in infalling field galaxies before they enter the cluster central regions where gas is stripped and star formation suppressed. Our results are also consistent with those of Porter \& Raychaudhury (2007), who have used optical data to discover an enhanced star-forming activity among galaxies associated with filaments in the nearby 
Pisces-Cetus supercluster. They also claim that the SFR in the filament galaxies peaks at 3-4 Mpc from the cluster center, which is consistent with our findings. However, we could better assess this result by extending our coverage further to the east.

Since our results are based on the study of a single cluster, it would be hazardous to generalize them to the entire cluster population, as different clusters may follow different evolutionary paths. With the completion of our observational program, another two clusters at $z \simeq 0.2$ will be analyzed to confirm that galaxy evolution is indeed speeding up in cluster-feeding filaments.

This work is based in part on observations made with Spitzer, a space telescope operated by the Jet Propulsion Laboratory, California Institute of Technology, under a contract with
NASA. Support for this work was provided by NASA through an award issued by JPL/Caltech. Funding for the SDSS and SDSS-II has been provided by the Alfred P. Sloan Foundation, the Participating Institutions, the National Science Foundation, the US Department of Energy, NASA, the Japanese Monbukagakusho, the Max Planck Society, and the Higher Education Funding Council for England. The SDSS is managed by the Astrophysical Research Consortium for the Participating Institutions (see list at http://www.sdss.org/collaboration/ credits.html).

Facilities: Spitzer (MIPS), Spitzer (IRAC), Palomar 200in (LFC), Palomar 200in (WIRC), WIYN (Hydra), TNG (Dolores).

\section{REFERENCES}

Bai, L., et al. 2006, ApJ, 639, 827 2007, ApJ, 664, 181

Balogh, M. L., Navarro, J. F., \& Morris, S. L. 2000, ApJ, 540, 113

Balogh, M. L., et al. 1998, ApJ, 504, L75

Barnes, J. E., \& Hernquist, L. 1996, ApJ, 471, 115

Barton, E. J., Geller, M. J., \& Kenyon, S. J. 2000, ApJ, 530, 660

Bekki, K. 1999, ApJ, 510, L15

Benson, A. J. 2005, MNRAS, 358, 551

Biviano, A., et al. 2004, A\&A, 425, 33

Carlberg, R. G., et al. 1997, ApJ, 485, L13

Ceccarelli, M. L., et al. 2005, ApJ, 622, 853

Cortese, L., et al. 2006, A\&A, 453, 847

de Lapparent, V., Geller, M. J., \& Huchra, J. P. 1986, ApJ, 302, L1

den Hartog, R., \& Katgert, P. 1996, MNRAS, 279, 349

Doroshkevich, A. G., et al. 2001, MNRAS, 322, 369

Dressler, A. 1980, ApJ, 236, 351

Fadda, D., Slezak, E., \& Bijaoui, A. 1998, A\&AS, 127, 335

Fasano, G., \& Franceschini, A. 1987, MNRAS, 225, 155

Fazio, G. G., et al. 2004, ApJS, 154, 10

Fujita, Y. 2004, PASJ, 56, 29

Geach, J. E., et al. 2006, ApJ, 649, 661

Gerken, B., et al. 2004, A\&A, 421, 59

Girardi, M., et al. 1996, ApJ, 457, 61

Goto, T., et al. 2003, MNRAS, 346, 601
Kennicutt, R. C., Jr. 1998, ARA\&A, 36, 189

Kodama, T., et al. 2004, MNRAS, 354, 1103

Lacy, M., et al. 2007, AJ, 133, 186

Lima-Neto, G., \& Durret, F. 2007, A\&A, submitted

Lin, Y.-T., Mohr, J. J., \& Stanford, S. A. 2003, ApJ, 591, 749

Mamon, G. 1996, in Third Paris Cosmology Colloquium, ed. H. J. de Vega \& N. Sanchez (Singapore: World Scientific), 95

Marcillac, D., et al. 2007, ApJ, 654, 825

Metcalfe, L., Fadda, D., \& Biviano, A. 2005, Space Sci. Rev., 119, 425

Navarro, J. F., Frenk, C. S., \& White, S. D. M. 1997, ApJ, 490, 493

Pimbblet, K. A., Drinkwater, M. J., \& Hawkrigg, M. C. 2004, MNRAS, 354, L61

Polletta, M., et al. 2007, ApJ, 663, 81

Porter, S. C., \& Raychaudhury, S. 2007, MNRAS, 375, 1409

Prokhorov, D. A., \& Durret, F. 2007, A\&A, 474, 375

Rieke, G. H., et al. 2004, ApJS, 154, 25

Rines, K., et al. 2005, AJ, 130, 1482

Silva, L., et al. 1998, ApJ, 509, 103

Skrutskie, M. F., et al. 2006, AJ, 131, 1163

Treu, T., et al. 2003, ApJ, 591, 53

Werner, M. W., et al. 2004, ApJS, 154, 1

Young, J. S., \& Scoville, N. Z. 1991, ARA\&A, 29, 581

Zabludoff, A. I., \& Franx, M. 1993, AJ, 106, 1314 